Research Paper

\title{
Bioimpedance Detection of Oral Lichen Planus Used as Preneoplastic Model
}

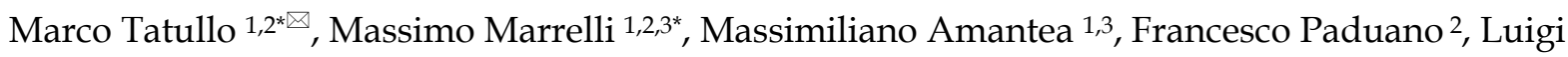 \\ Santacroce ${ }^{4}$, Stefano Gentile $2,3^{*}$, Salvatore Scacco ${ }^{5^{*}}$ \\ 1. Unit of Maxillofacial Surgery, Calabrodental, Crotone, Italy \\ 2. Tecnologica Research Institute, Biomedical Section, Crotone, Italy \\ 3. Marrelli Hospital, Cancer Surgery Unit, Crotone, Italy \\ 4. Dept. JSGEM, University of Bari - Section of Taranto, Italy \\ 5. Dept. of Basic Medical Sciences, Neurosciences and Sense Organs, University of Bari, Italy \\ * These Authors equally contributed to this research \\ $\triangle$ Corresponding author: Dr. Marco Tatullo, PhD, Scientific Director - Tecnologica Research Institute, St. E. Fermi - Crotone, Italy. Phone: \\ +390962930362; Mobile: +393498742445; E-mail: marco.tatullo@tecnologicasrl.com
}

(C) 2015 Ivyspring International Publisher. Reproduction is permitted for personal, noncommercial use, provided that the article is in whole, unmodified, and properly cited. See http://ivyspring.com/terms for terms and conditions.

Received: 2015.02.19; Accepted: 2015.06.11; Published: 2015.08.20

\begin{abstract}
Introduction: Bioimpedance is a measure of the electrical properties of biological tissues. In the last two decades bioimpedance has been successfully introduced in clinical diagnosis of cancer. It has been demonstrated that tumoral tissues often show lower bioimpedance values than healthy tissues.

The aim of this work is to assess the bioimpedentiometric differences between healthy and Oral Lichen Planus (OLP) affected oral mucosa, taking attention to the erosive form which may represent a potential pre-cancerous condition.

Methods: 52 patients affected by OLP were recruited for bioimpedance examination of oral mucosa. Four electrical properties, resistance $(R)$, reactance $\left(X_{c}\right)$, phase angle $(\theta)$ and impedance $(Z)$ of the tongue and of the intraoral mucosa, were measured.

Results: We observed a significant increase of $Z$ and a significant decrease of $\theta$ values in correspondence of OLP lesions compared to healthy oral mucosa, and a marked decrease of $Z$ values in correspondence of erosive OLP lesions.

Conclusions: These results provide evidence of the usefulness of bioimpedance assay for the characterization of healthy and clinically OLP affected mucosa. Bioimpedance is a valid aid in the early detection and clinical monitoring of the suspicious lesions which could lead to a potentially malignant evolution.

The present research article is a valuable addition to the scientific literature of cancer prevention, and our findings can be considered extremely encouraging as they represent the initial step for a more wide clinical study for better define the different cut-off values in the different precancerous conditions occurring in the oral mucosa.
\end{abstract}

Key words: Oral Pathology, Early Diagnosis, Oral Cancer, Cancer Prevention

\section{Introduction}

Bioimpedance is the ability of biological tissues to impede electric current ${ }^{1}$. Depending on the source of electricity, it is possible to distinguish two main different responses: an active response occurs when biological tissues provoke electricity from ionic activities inside the cells, such as in electrocardiograph signals, or electroencephalograph signals; instead, passive response occurs when tissues are stimulated 
through an external current source ${ }^{2}$. The analysis of bioimpedance signals can give useful informations about the electrochemical processes occurring in the target tissue, thus allowing a constant monitoring of any physio-pathological change. Depending on the frequency of the applied electric field, we can observe different dielectric dispersions listed as a$(10 \mathrm{~Hz}-10 \mathrm{kHz}), \quad \beta-(10 \mathrm{kHz}-10 \mathrm{MHz})$ and $\gamma^{-}$(over $10 \mathrm{MHz})^{3}$. The $\alpha-$ and $\beta$-dispersion regions emerged as the more interesting in medical applications ${ }^{4}$; they are respectively related to the ionic environment surrounding the cells and to the tissue structure relaxation ${ }^{1}$. Due to the noninvasiveness, the reliability, the immediacy of the results, the low cost and the portability of the whole system, many researchers conducted studies on bioimpedance and its application in common clinical diagnostic procedures ${ }^{5}$.

The preliminary applications of bioimpedance assay were focused to the early detection of breast cancer in the $90 \mathrm{~s}{ }^{6}$ they were later improved and successfully tested as useful aid for the clinical diagnosis of cervical cancer ${ }^{7}$ and breast cancer ${ }^{8}$.

Although it represents only the $2 \%$ of all the yearly diagnosed cancers 9,10 , oral squamous cell carcinoma (OSCC) exhibits a higher proliferating rate compared to the more rare odontogenic tumors 11 , furthermore, it has the highest incidence among oral mucosa malignancies 12 ; the easiness of the interaction between oral mucosa and several pro-oncogenetic chemicals such as smoke-related metabolites ${ }^{13}$, or with oncogenic viruses and other well known factors ${ }^{14}$, is on the other hand followed by a remarkable difficulty to early detect the dysplastic lesions, often incorrectly diagnosed as aspecific inflammatory manifestations or, less frequently, unrecognized as early pre-neoplastic lesions ${ }^{15}$.

In this light, the improvement of a noninvasive screening for the potential pre-cancerous lesions, as it could happen for any chronic diseases affecting the oral mucosa, represents a useful aid for the early detection of oral cancer in its preliminary stages.

OLP is a chronic inflammatory disease affecting the oral mucosa, with a prevalence of about $1-2 \%$ of the population ${ }^{16}$. The histological picture of OLP is characterized by a dense sub-epithelial lymphocytes infiltration, increased number of intraepithelial lymphocytes, and the degeneration of basal keratinocytes. The lymphocytic infiltrates consists predominantly of T-cells (CD4 and CD8) 17,18.

OLP can manifest itself in different clinical forms: the keratotic, reticular and erosive, which usually occur bilaterally on the oral mucosa and more rarely on lateral borders of the tongue and gums ${ }^{19}$. Once arisen, the lesions unlikely undergo to self-remission ${ }^{18}$ and, in the $6.5 \%$ of cases, some of them have the ability to show a malignant behavior ${ }^{20,}$ leading to OSCC of the oral epithelium ${ }^{17}$. In view of these findings, the World Health Organization has classified OLP as one of the oral potentially malignant diseases (OPMDs) ${ }^{17}$.

Based on these assumptions, and considering that the promptness of the diagnosis represents the most critical aspect in the cancer prevention and treatment ${ }^{14,21}$, the aim of the present work is to evaluate and to validate, by using a bioimpedance analyzer, the efficiency of bio-electrical impedance analysis as a new, noninvasive and real-time screening diagnostic method to assess the electrical properties of oral mucosa affected by OLP; our main goal is to set up the bioimpedance values which characterize the reticular and the erosive forms of OLP, and to early detect those suggestive forms of potentially dysplastic lesions which may occur on OLP affected mucosa.

This work will contribute to design a standardized protocol for the monitoring of the sites into oral cavity with a higher risk of developing oral cancer. In this study we have also used for the first time a new customized prototype probe, able to detect the bioimpedance values of the oral mucosa.

\section{Methods}

\section{Study Design}

This cross/sectional study was conducted in collaboration between Calabrodental dental clinic (Crotone, Italy) and the University of Bari (Italy), jointly involved in the Ne.T.T.Un.O (The NEw Technologies used in the diagnosis and the Treatment of UNclear lesions developed on the Oral tissues) project.

All the measurements were performed on adult patients ( $54 \pm 11$ years), according with the informed consent guidelines approved by the Calabrodental dental clinic (Crotone, Italy). The Calabrodental Ethics Committee specifically approved this study and its related procedures (Prot. July-2012/Res005). Written informed consent was obtained from the participants of the study using a specific form. The study followed the "Ethical principles for medical research involving human subjects" of the Helsinki Declaration.

\section{Patients}

We recruited 52 patients (29\% male, $71 \%$ female, mean age $54 \pm 11$ years) affected by OLP, and 11 healthy patients as a control, in Calabrodental clinic. In 18 of the 52 OLP patients, the lesions were on the tongue; 34 patients showed reticular OLP lesions onset on the intraoral mucosa among these, eight of them developed hyperkeratotic plaques. Six of them were previously treated for reticular OLP lesions, administering the topical treatment with steroids, as 
reported in the literature 18 , only three patients out of 34 were diagnosed a severe form of OLP with erosive lesions. Clinical and histopathological diagnosis was carried out according with the most recent guidelines previously described ${ }^{22}$.

Each patient gave signed informed consent, accordingly to the Declaration of Helsinki, Ethical Principles for Medical Research Involving Human Subjects Committee, and Italian laws on the use of human subjects for medical research.

\section{Bioimpedance measurement}

Every measurement has been performed in the Oral Pathology Unit of Calabrodental clinic by the same clinician. The bioimpedance analyzer used in this research was a medical device, approved by European Community as Class IIA-Type BF-IMQ SPA, conformed to the following European regulations: 2007/47/CEE-EN606011-2, EN60601-1.

The prototype probe used in the present work was designed to create a homogeneous electric field with the four-electrodes innovative design: this particular disposition enables to apply a high-frequency alternating current through the external electrodes on the biological tissue, while the middle electrodes relieve the intensity and the delay (voltage and phase) of the electric field generated (Figure 1).

The gap between each electrode has been optimized for measurements at $50 \mathrm{kHz}$ frequency, which allows for analyze electrical properties of biological tissues up to $2 \mathrm{~cm}$ depth, with surfaces having impedance at contact of $\leq 200 \mathrm{ohm}$, and with a global sample impedance of $400 \mathrm{ohm}$.
As previously stated, many studies indicated at $50 \mathrm{kHz}$ the frequency able to guarantee the higher reproducibility of bioimpedance measurements ${ }^{1}$.

The probe has a small head with 4 electrodes, a minimally displaceable neck and a rigid part as a handgrip: this probe was placed on OLP lesions as and on healthy areas of the tongue and of the intraoral mucosa, thanks to its small size (Figure 2). The four electrical impedance parameters measured and archived were: $R$, which represents the pure opposition of a biological conductor to the flow of an alternating electric current; $X_{c}$, representing the resistive effect due to capacitance produced by tissue interfaces and cell membranes; $\theta$, that reflects the relative contributions of fluid (resistance) and cellular membrane.

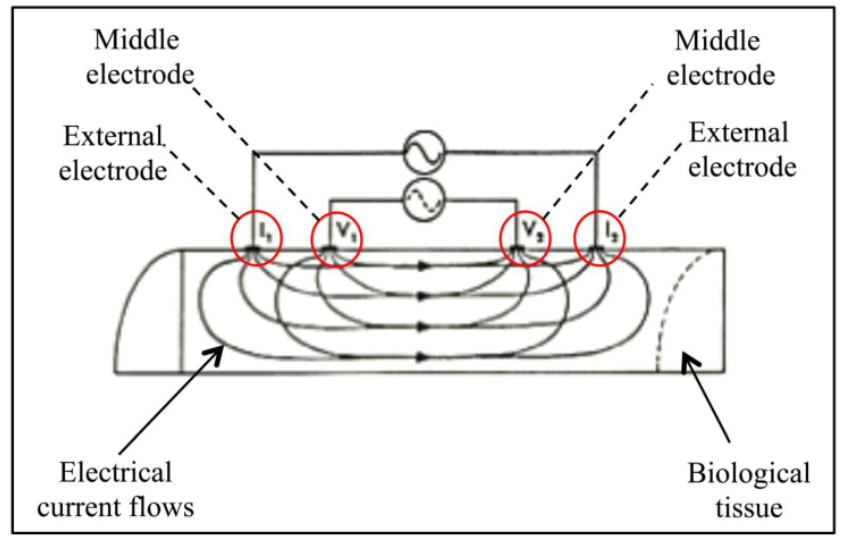

Figure 1. SCHEMATIC REPRESENTATION OF THE PROBE FUNCTIONING MECHANISM. The alternating current flows through external electrodes (II and I2); the middle electrodes (VI and V2) relieve the delay and the intensity of the electric current that passes through the biological tissue.

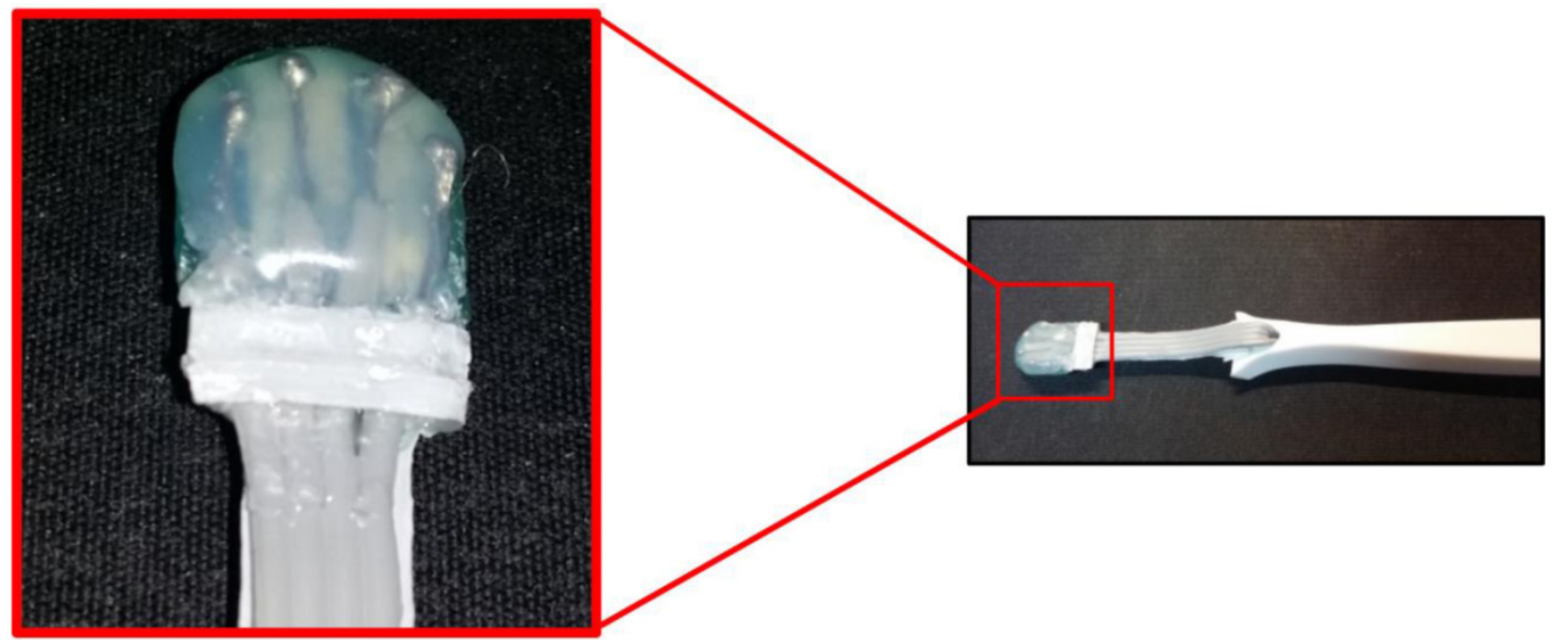

Figure 2. THE INTRAORAL PROBE. A view of the prototype probe with in evidence the "head" containing the four micro-electrodes, placed at 2 mm of distance each other and with a total width of $1 \mathrm{~cm}$. 


\section{Outcomes variability}

The probe accuracy was evaluated by the manufacturing company and resulted to be $\pm 0.1 \mathrm{ohm}$. All the measurements have been performed in the same conditions and by the same clinician, the corresponding author of this paper, while environment and patient intraoral temperatures were constantly monitored throughout the duration of the entire procedure. We used dry sterile gauzes right before placing the prototype probe on the tongue and on the intraoral mucosa of each patient, to prevent possible alterations of the outcomes, due to excessive salivation. All these expedients were taken to minimize the potential sources of bias.

\section{Statistical Analysis}

Each value was expressed as the mean \pm standard deviation, out of 3 measurements per patient. Paired-sample t-test was used to determine whether there were significant differences of electrical impedance between healthy tissue and OLP lesions, and between reticular and erosive OLP lesions developed on the oral mucosa. Variations with $\mathrm{p} \leq 0.05$ were considered statistically significant. To verify the sensitivity and the specificity of the method used in this work, a ROC curve (Receiver Operating Characteristic curve) was created, as previously described ${ }^{23}$.

\section{Results}

\section{Patients with OLP onset on the tongue}

In the patients with OLP lesions onset on the tongue, we observed a significant increase of $\mathrm{R}$ and $\mathrm{Xc}_{\mathrm{c}}$ values between the area of the tongue affected by OLP, compared to the healthy group investigated (see Supplementary Table 1 ). As a consequence, $Z$ values resulted significantly increased $(p=0.02)$ while, on the other hand, $\theta$ values decreased $(p=0.04)$ (Table 1).

Table 1. aHT= healthy tongue; $\mathrm{TLs}=$ oral lichen planus lesions onset on the tongue; $\mathrm{HM}=$ healthy intraoral mucosa; $\mathrm{RLs}=$ reticular oral lichen planus lesions onset on the intraoral mucosa; $\mathrm{HPs}=$ hyperkeratotic plaques onset on the intraoral mucosa; $\mathrm{Tr}=$ patients treated for reticular oral lichen planus lesions; ELs= erosive oral lichen planus lesions onset on the intraoral mucosa. Values are expressed as mean \pm standard deviation.

\begin{tabular}{lll}
\hline & $\mathbf{Z} \mathbf{( o h m )}$ & $\boldsymbol{\theta}(\mathbf{d e g})$ \\
\hline HTa & $806 \pm 52.9$ & $29.5 \pm 3.3$ \\
TLs $^{a}$ & $1650.3 \pm 74$ & $16 \pm 4$ \\
HMa & $1224.4 \pm 155$ & $30.9 \pm 4$ \\
RLs $^{a}$ & $1740.5 \pm 50.8$ & $19.6 \pm 3$ \\
HPs $^{a}$ & $2060.5 \pm 103.6$ & $23 \pm 4.4$ \\
Tra $^{a}$ & $1445 \pm 101.3$ & $32 \pm 2.7$ \\
ELs $^{\mathrm{a}}$ & $913.7 \pm 55.8$ & $26 \pm 4$ \\
\hline
\end{tabular}

\section{Patients with reticular OLP lesions onset on the intraoral mucosa}

Similarly, the $\mathrm{R}$ and $\mathrm{Xc}_{\mathrm{c}}$ values measured in patients with OLP lesions on the intraoral mucosa, were both significantly higher compared to the healthy mucosa, $Z$ values resulted significantly increased $(p=0.01)$ and $\theta$ values significantly decreased $(p=0.01)$ (Table 1). We further noticed that patients with OLP lesions on the intraoral mucosa who developed hyperkeratotic plaques were characterized by markedly high values of $Z$ ( $p=0.005)$, compared to the healthy mucosa; moreover, they showed a significant decrease of $\theta$ values $(\mathrm{p}=0.05)$ (Table 1$)$.

\section{Patients previously treated for OLP lesions onset on the intraoral mucosa}

In those patients previously treated for OLP lesions, we observed a slight, not statistically significant, increase of $Z$ values with respect to the surrounding healthy mucosa (Table 1 ), while the $\theta$ values were quite similar to the values of the healthy mucosa (Table 1).

\section{Patients with erosive OLP lesions onset on the intraoral mucosa}

Three patients showed erosive lesions and their $\mathrm{Z}$ values appeared strongly decreased if compared to those measured in correspondence of reticular lesions $(p=0.01)$ and hyperkeratotic plaques $(p=0.02)$ of the other patients (Table 1). However, the most intriguing data is the decrease of $\mathrm{Z}$ values observed between erosive lesions and healthy mucosa (Table 1). No significant variations of $\theta$ values were observed in this specific case (Table $\mathbf{1}$ ).

\section{Evaluation of the sensitivity of bioimpedance assay}

Furthermore, according to the results above described, we spotted different ranges of $\mathrm{Z}$ values, which indicate the electrical peculiarities characterizing the different forms of OLP lesions occurring on the oral mucosa. We indicated these ranges in Table 2 and we represented them graphically in Figure 3, with a color-code scale associated. Finally, the validity and the sensitivity of our bioimpedance model, as a good predictor of different forms of OLP lesions, were confirmed by the evaluation of the area under the ROC curve (AUC=0.89), as indicated in Table 2. 

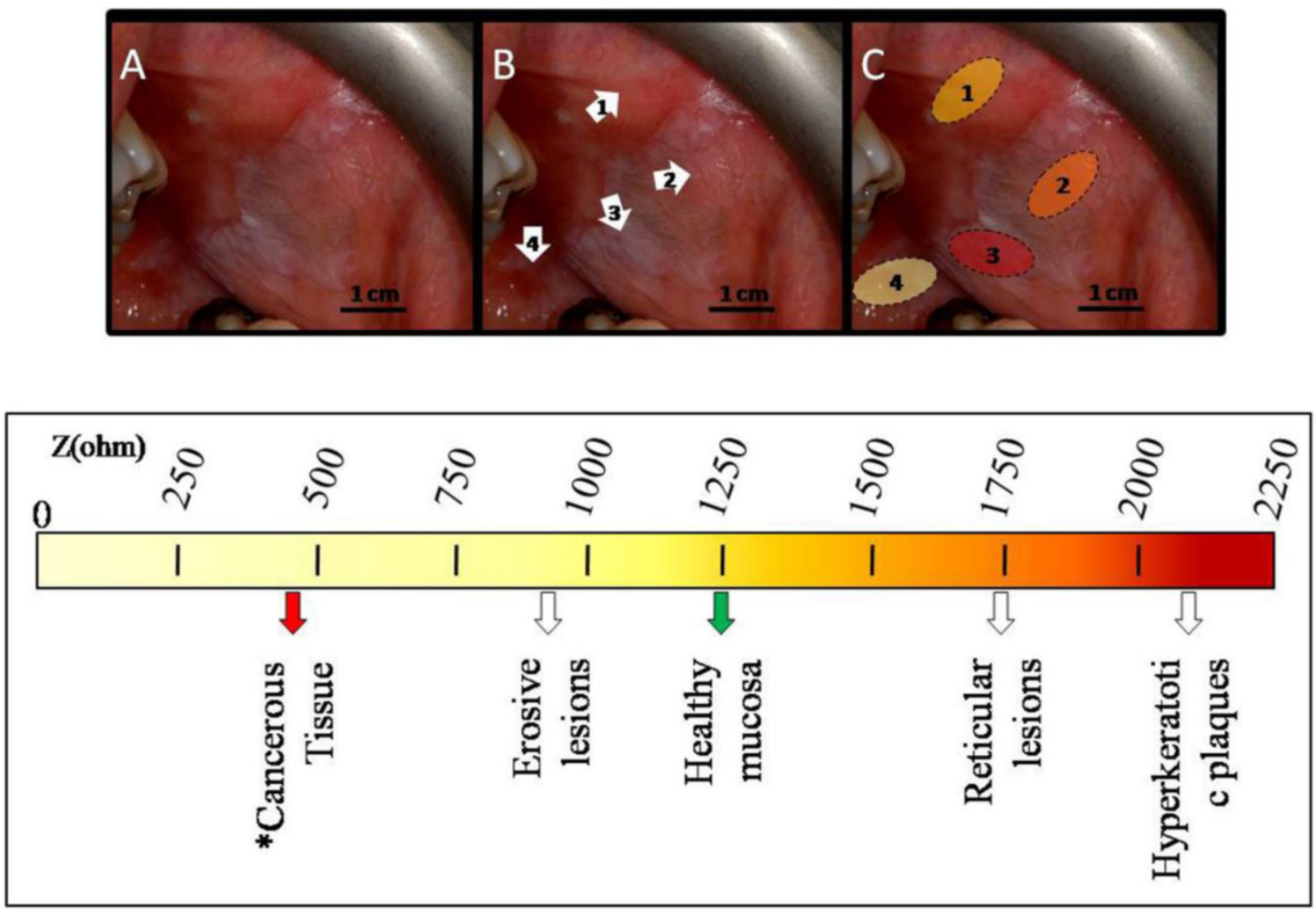

Figure 3. BIOIMPEDANCE LIMIT VALUES FOR OLP LESIONS NEOPLASTIC POTENTIAL. A: patient affected by OLP on the intraoral mucosa; B: white arrows indicate 1) healthy oral mucosa, 2) reticular OLP lesions, 3) hyperkeratotic plaques, 4) erosive OLP lesions; C: the hatched areas correspond to different portions of the intraoral mucosa affected by reticular (2), hyperkeratotic (3) and erosive (4) OLP lesions. The colors represent the intensity of the $Z$ value registered in correspondence of each specific area as indicated above. On the chromatic scale below are reported the $Z$ values observed for each OLP lesion analyzed in the present study, as well as for healthy intraoral mucosa. We also added the $Z$ value reported for cancerous tongue tissue by Ching et al. '.

Table 2. In the second column are indicated the ranges of $Z$ values which characterize different forms of OLP lesions; in the third column are reported the percentages of diagnosed OLP on the intraoral mucosa among all patient visited in the present work ( $N=52)$. The Area under the ROC curve $(A \cup C=0.89)$ indicates the level of sensitivity and specificity of bioimpedance analysis in diagnosing OLP lesions at different stages.

\begin{tabular}{lll}
\hline Intraoral Mucosa & Electric Impedance (Z) ranges & \% of diagnosed cases (N=52) \\
\hline Healthy Oral Mucosa & $1070-1380 \mathrm{ohm}$ & \\
Erosive OLP lesions & $850-970 \mathrm{ohm}$ & $5.8 \%$ \\
Reticular OLP lesions & $1690-1790 \mathrm{ohm}$ & $32.7 \%$ \\
Hyperkeratotic Plaques & $1960-2160 \mathrm{ohm}$ & $15.4 \%$ \\
Receiver Operating Characteristic (ROC) curve & &
\end{tabular}

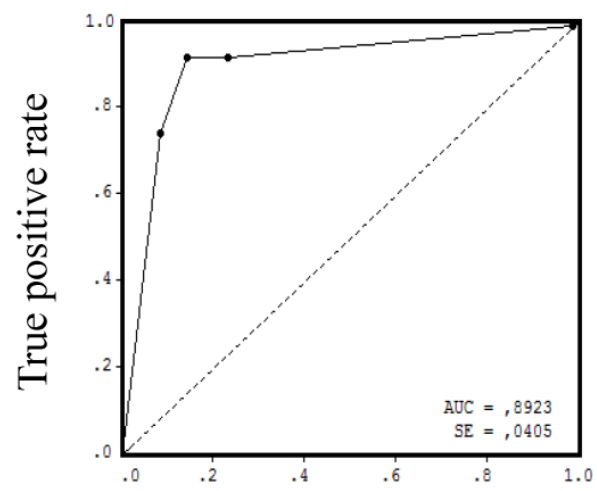

False positive rate 


\section{Discussion}

In the present study we used a commonly commercially bioimpedance analyzer to assess the bioimpedance values which may characterize the electrical properties of the different forms of OLP lesions and, particularly, of the OLP erosive form, a well known potentially pre-cancerous condition ${ }^{17}$.

The bioimpedance $\mathrm{Z}$ parameter showed an overall significant increase in correspondence of OLP lesions, when compared to the surrounding healthy mucosa. This could be explained by considering the histological features of OLP lesions that usually show a dense inflammatory infiltrate in the upper lamina propria, as well as high degeneration of basal keratinocytes and hyperkeratosis 17, 18. Recently, a study carried out on skin keratotic lesions, the Clavus, hypothesized that the relative high bioimpedance values observed may be due to the massive hyperkeratosis, typical of Clavus lesions ${ }^{23}$. In our experience, the hyperkeratosis induced by OLP 18,24 could be responsible of the increase of $Z$ values registered in correspondence of these lesions, compared to the healthy surrounding mucosa.

The other main electrical parameter, the phase angle $(\theta)$, appeared significantly decreased when measured on OLP lesions, with respect to the healthy mucosa. These data are consistent with the existing literature about the biological significance of $\theta$, which represents the measure of the relation between $X_{c}$ and $\mathrm{R}{ }^{25}$. By definition, $\theta$ is positively associated with $X_{c}$, which can be considered as a measure of the volume of cell membranes capacitance, and negatively associated with $\mathrm{R}$, which is determined by the extracellular fluids ${ }^{26}$. Reduced cell integrity or cell necrosis is typical with low $\theta$ values; on the contrary, huge quantities of intact cell membranes may be indicated by high $\theta$ values ${ }^{26}$. In our case, the significantly low $\theta$ values observed may be related to the inflammatory cells-mediated killing of keratinocytes. Although several molecular studies have indicated an enhanced turnover rate in the epithelial cells within reticular OLP lesions ${ }^{27}$, our findings seem to be more in accordance with other authors which reported mixed patterns of apoptosis or, in some specific cases, the presence of keratinocytes showing a senescent phenotype, occurring simultaneously with cells proliferation 27.

In patients that were properly treated for OLP lesions, though they didn't show any clinical evidence of the disease after conventional oral examination (COE), the $Z$ and $\theta$ values of their oral mucosa appeared to be halfway between those observed for healthy mucosa, and those showed by patients affected by OLP lesions.
Lastly, $\mathrm{Z}$ values measured on the erosive lesions were significantly lower with respect to the values previously registered for reticular lesions and for hyperkeratotic plaques. Moreover, in patients with erosive OLP the $Z$ values measured on erosive lesions were suggestively lower, if compared to healthy surrounding mucosa. These results appear to be consistent with Ching et al. observations ${ }^{1}$; they measured the electrical impedance properties of both cancerous and normal tissue in such patients affected by tongue cancer. In their study, $\mathrm{Z}$ resulted significantly lower in cancerous tissue than in healthy tissue, as also reported in literature by other authors 6,28. The decrease of $Z$ values on cancerous tissue could be related to the loss of the physiological cell-to-cell interactions, as consequence of the phenomenon of metastasis, which causes an impairment of tissue packing, hence offering low resistance to electrical currents 1 .

In our study, the inflammatory processes underlying the onset of erosive OLP could have induced a strong degradation of the keratinocytes layer, typical of erosive lesions where lymphocytes destructive action overcomes epithelial regenerative reaction ${ }^{18}$.

Even if the triggering mechanism that induces the transformation of OLP lesions into neoplastic lesions is still unclear, the severe erosive forms of OLP are reported to carry the highest risk of malignant transformation ${ }^{17}$. The current hypothesis is that the chronic stimulation promoted by the inflammatory cells can provide the signals that induce epithelial cells to an uncontrolled growth: this condition, in cooperation with oxidative damage, would provoke DNA alteration resulting in neoplastic transformation $^{19}$.

In the light of the above findings, our results may provide some evidences on the usefulness and the reliability of bioimpedance analysis as a diagnostic tool for the different forms of OLP. This method has demonstrated to provide reference values of the bioimpedance able to distinguish the classic reticular lesions from the hyperkeratotic plaques and from the erosive lesions; moreover, our study has assessed the limit values representing a dangerous alarm bell for the possible neoplastic evolution of OLP.

In conclusion, our study was aimed to improve the diagnostic capabilities of the clinicians, given that many of human tumors develop their growth for months, or even years, before they become clinically detectable, and lots of them could metastasize even before the primary tumor is diagnosed ${ }^{29}$. The challenge, therefore, is to advance the oncological diagnosis to an earlier stage thanks to a new method for reliable, low-cost, noninvasive and real-time screening of cancer detection ${ }^{30,31}$. 
We believe that bioimpedance analysis could represent the first step towards the creation of a standardized protocol for the periodical monitoring of OLP lesions and for the identification of those erosive lesions which can be potentially pre-cancerous. In fact, this method can be easily used in the clinical practice because it surely induces less anxiety in patients with respect of a common surgical biopsy, although not substituting for it. We are planning also to test this new technique on other sites, such as vaginal mucosa or esophageal mucosa: every anatomical area will be tested with a newly designed and developed probe, closely customized to the peculiarity of the area to be investigated.

The challenge is to characterize all the basal and altered values of the different anatomical sites: based on these data, we could have a clear indication of those lesions that should be better investigated, based on the detected bioimpedance values.

Finally, our goal is to further test this methodology with other potentially pre-neoplastic conditions, so as to create a diriment panel of the different ranges of values, closely related to the different clinical conditions.

\section{Supplementary Material}

Supplementary Table 1.

http://www.jcancer.org/v06p0976s1.pdf

\section{Acknowledgments}

The present study was performed in the "Ne.T.T.Un.O. Project - NEw Technologies used in the diagnosis and the treatment of UNclear lesions developed on the Oral tissues" developed in collaboration between the University of Bari, Dept. of Basic Medical Science, and Calabrodental SRL.

We are grateful to Dr. Ernesto Chiaravalloti, Dr. Nicola Sardaro and all the Dept. of Basic Medical Sciences for their collaboration in this research project.

The prototypal probe was conceived and designed by Eng. Antonio Talluri, based on specific requests and suggestions by Calabrodental SRL. We thank Eng. Talluri and AKERN Srl for the high-quality work.

The revised prototypal probe was conceived and designed by Dr. Marco Tatullo and Dr. Stefano Gentile.

We are grateful to Concetta Stanizzi, Esq., and L.I.L.T. (Lega Italiana Lotta ai Tumori), especially the seat of Catanzaro, for their support to this study and for their high contribution to the fight against cancer.

\section{Conflict of Interest}

All authors declare to have no conflict of interest.

\section{References}

1. Ching CT, Sun TP, Huang SH, Hsiao CS, Chang CH, Huang SY et al. A preliminary study of the use of bioimpedance in the screening of squamous tongue cancer. International Journal of Nanomedicine. 2010; 5:213-20.

2. Kyle UG, Bosaeus I, De Lorenzo AD, Deurenberg P, Elia M, Gomez JM et al.. Bioelectrical impedance analysis Part 1: review of principles and methods. Clinical Nutrition. 2004; 23:1226-43.

3. Abdalla S. Low frequency dielectric properties of human blood. IEEE Transaction on Nanobioscience. 2011; 10:113-20.

4. Jahnke HG, Heimann A, Azendorf R, Mpoukouvalas K, Kempski O, Robitzki AA et al. Impedance spectroscopy: an outstanding method for label-free and real-time discrimination between brain and tumor tissue in vivo. Biosensors and Bioelectronics. 2013; 46:8-14.

5. Bayford R, Tizzard A. Bioimpedance imaging: an overview of potential clinical applications. Analyst. 2012; 137:4635-43.

6. Morimoto T, Kimura S, Konishi Y, Komaki K, Uyama T, Monden $\mathrm{Y}$ et al. A study of the electrical bioimpedance of tumors. Journal of Investigative Surgery. $1993 ; 6: 25-32$

7. Brown BH, Tidy JA, Boston K, Blackett AD, Smallwood RH, Sharp F. Relation between tissue structure and imposed electrical current flow in cervical neoplasia. Lancet. 2000; 355:892-5.

8. Stojadinovic A, Nissan A, Gallimidi Z, Lenington S, Logan W, Zuley M et al. Electrical impedance scanning for the early detection of breast cancer in young women: preliminary results of a multicenter prospective clinical trial. Journal of Clinical Oncology. 2005; 23:2703-15.

9. Jemal A, Bray F, Center MM, Ferlay J, Ward E, Forman D. Global cancer statistics. CA Cancer Journal for Clinicians. 2011; 61:69-90.

10. Siegel R, Naishadham D, Jemal A. Cancer statistics, 2012. CA Cancer Journal for Clinicians. 2012; 62:10-29.

11. Marrelli M, Pacifici A, Di Giorgio G, Cassetta M, Stefanelli LV, Gargari M et al. Diagnosis and treatment of a rare case of adenomatoid ondontogenic tumor in a young patient affected by attenuated familial adenomatosis polyposis (aFAP): case report and 5 year follow-up. European Review for Medical and Pharmacological Sciences. 2014; 18:265-9.

12. Laeijendecker $R$, Van Joost $T$, Kuizinga MC, Tank B, Neumann HA. Premalignant nature of oral lichen planus. Acta Dermato-Venereologica. 2005; 85:516-20

13. Ferreira Antunes JL, Toporcov TN, Biazevic MG, Boing AF, Scully C, Petti S. Joint and independent effects of alcohol drinking and tobacco smoking on oral cancer: a large case-control study. PLoS One. 2013; 8:e68132.

14. Epstein JB, Thariat J, Bensadoun RJ, Barasch A, Murphy BA, Kolnick L et al.). Oral complications of cancer and cancer therapy: from cancer treatment to survivorship. CA Cancer Journal for Clinicians. 2012; 62:400-22

15. van der Waal I, de Bree R, Brakenhoff R, Coebergh JW. Early diagnosis in primary oral cancer: is it possible? Medicina Oral Patologia Oral y Cirurgia Bucal. 2011; 16:e300-5.

16. Bajpai M, Agarwal D, Bhalla A, VatchalaRani RM, Kumar M. Unilateral lichen planus: a rare case report. Journal Natural Science Biology and Medicine. 2014; 5:453-5.

17. Georgakopoulou EA, Achtari MD, Achtaris M, Foukas PG, Kotsinas A. Oral lichen planus as a preneoplastic inflammatory model. J Biomedicine and Biotechnology. 2012; 2012:759626.

18. Di Stasio D, Guida A, Salerno C, Contaldo M, Esposito V, Laino L et al. Oral lichen planus: a narrative review. Frontiers in Bioscience (Elite Ed.). 2014; 6:370-376.

19. Gonzalez-Moles MA, Scully C, Gil-Montoya JA. Oral lichen planus: controversies surrounding malignant transformation. Oral Diseases. 2008; 14:229-43.

20. Redder CP, Pandit S, Desai D, Kandagal VS, Ingaleshwar PS, Shetty SJ et al. 2014. Comparative analysis of cell proliferation ratio in plaque and erosive oral lichen planus: an immunohistochemical study. Dental Research Journal. 2014; 11:316-20.

21. Kalpathy-Cramer J, Gerstner ER, Emblem KE, Andronesi OC, Rosen B. Advanced magnetic resonance imaging of the physical processes in human glioblastoma. Cancer Research. 2014; 74:4622-37.

22. Khudhur AS, Di Zenzo G, Carrozzo M. Oral lichenoid tissue reactions: diagnosis and classification. Expert Review of Molecular Diagnostics. 2014; 14:169-84.

23. Hung CY, Sun PL, Chiang SJ, Jaw FS. In vitro differential diagnosis of clavus and verruca by predictive model generated from electrical impedance. PLoS One. 2014; 9:e93647.

24. Alessi SS, Nico MM, Fernandes JD, Lourenco SV. Reflectance confocal microscopy as a new tool in the in vivo evaluation of desquamative gingivitis: patterns in mucous membrane pemphigoid, pemphigus vulgaris and oral lichen planus. British Journal of Dermatology. 2013; 168:257-64.

25. Malecka-Massalska T, Chara K, Golebiowski P, Wladysiuk M, Smolen A, Kurylcio A et al. Altered electrical properties in women with breast cancer: preliminary observations. Annals of Agriculture and Environmental Medicine. 2013; 20:523-7.

26. Gupta D, Lis CG, Dahlk SL, Vashi PG, Grutsch JF, Lammersfeld CA. Bioelectrical impedance phase angle as a prognostic indicator in advanced pancreatic cancer. British Journal of Nutrition. 2004; 92:957-62.

27. Gupta D, Lammersfeld CA, Burrows JL, Dahlk SL, Vashi PG, Grutsch JF et al. Bioelectrical impedance phase angle in clinical practice: implications for 
prognosis in advanced colorectal cancer. American Journal of Clinical Nutrition. 2004; 80:1634-8.

28. Keshtkar A, Salehnia Z, Somi MH, Eftekharsadat AT. Some early results related to electric impedance of normal and abnormal gastric tissue. Physica Medica. 2012; 28:19-24.

29. Vogelstein B, Papadopoulos N, Velculescu VE, Zhou S, Diaz LA Jr, Kinzler KW. Cancer genome landscapes. Science. 2013; 339:1546-58.

30. Vineis P, Wild CP. Global cancer patterns: causes and prevention. Lancet. 2014; 383:549-57.

31. Chalkidou K, Marquez P, Dhillon PK, Teerawattananon $Y$, Anothaisintawee T, Gadelha CA et al. Evidence-informed frameworks for cost-effective cancer care and prevention in low, middle, and high-income countries. Lancet Oncolology. 2014; 15:e119-31. 